\title{
Electrolytes Status in Different Type of Acute Stroke Patients and Their Correlation with Some Common Clinical Presentation
}

\author{
MAHMUDUR RAHMAN SIDDIQUI, ${ }^{1}$ QUAZI TARIKUL ISLAM ${ }^{2}{ }^{2}$ MD AZHARUL HAQUE, ${ }^{3}$ MD JABED IQBAL, ${ }^{4}$ AHMED \\ HOSSAIN, ${ }^{5}$ YOUSUF UR RAHMAN, ${ }^{6}$ MD. SHAHRIAR MAHBUB, ${ }^{7}$ ASIF ALAM SAZZAD ${ }^{8}$
}

\begin{abstract}
:
Background: In our country, there are many studies on stroke, its associated conditions and their effect on stroke patient's outcome, but a few studies on electrolytes status in stroke patients have been done in our country, even outside.

Method: A total number of 100 randomly selected, clinically and CT proven acute stroke patients were studied at medicine units of Dhaka Medical College Hospital. Electrolytes status in different type of acute stroke patients and their association with some common clinical presentation were identified and correlated.

Result: Out of 100 patients, $29 \%$ were in between $51-60$ years age group \& $72 \%$ were male and $28 \%$ were female patients. Majority 53\% patients had Ischaemic stroke, $45 \%$ Intracerebral haemorrhage (ICH) and only $2 \%$ had Subarachnoid haemorrhage (SAH). 53\% of total acute stroke patient had dyselectrolytaemia. Among 100 acute stroke patients, $66.66 \%$ haemorrhagic stroke and $28.30 \%$ ischaemic stroke patients had headache $(p<0.05)$. $70.66 \%$ haemorrhagic and $30.23 \%$ ischaemic stroke patients had vomiting $(p<0.05) .53 \%$ of total acute stroke patient had dyselectrolytaemia. Among 100 acute stroke patient 49 (49\%) presented with vomiting from which $73.46 \%$ had dyselectrolytaemia $(p<0.01) .54$ (54\%) presented with headache from which $74.07 \%$ had dyselectrolytaemia $(p<0.001) .62 .22 \%$ of haemorrhagic stroke $(p<0.05) \& 43.39 \%$ of ischaemic stroke $(p>0.05)$ patients had dyselectrolytaemia. Total $36 \%$ of all stroke patients had serum sodium imbalance \& $31 \%$ had serum potassium imbalance. In haemorrhagic stroke \& ischaemic stroke patients, hyponatraemia (17\% \& 13\%), hypernatraemia (I\% \& 3\%), hypokalaemia ( I 9\% \& I I\%), hyperkalaemia (0\% \& I\%), hypochloraemia (9\% \& 6\%) respectively.
\end{abstract}

Conclusion: In haemorrhagic stroke, the incidence of electrolytes imbalance was more than ischaemic stroke and which were mostly hyponatraemia and hypokalaemia.

Keyword: Stroke, dyselectrolytaemia, electrolytes imbalance,

\section{Introduction:}

Stroke is the most common neurological emergency. ${ }^{1}$ Stroke is the third most common cause of death in developed nations after ischaemic heart disease and cancer. ${ }^{2}$ The incidence of stroke increases day by day and in many developing

1. Consultant, Medicine

2. Professor, Dept. of Medicine, DMC.

3. Associate Professor, Dept. of Neurology, DMC.

4. Student, FCPS Medicine P-II Course, DMC.

5. Assistant professor, Dept. of Medicine, DMC.

6. Student, FCPS Medicine P-II Course, DMC.

7. Assistant register, Dept. of Neurology, DMCH.

8. Intern medical officer, Dept. of Medicine, DMCH.

Correspondence : Dr. Mahmudur Rahman Siddiqui, Consultant Medicine, Dhaka, Bangladesh. E-mail: dr.mahmud99@yahoo.com countries, the incidence is rising because of adaptation of unhealthy life style and lack of awareness. ${ }^{2}$ The AHA estimates that 780000 strokes occur each year; 600000 of these are new strokes, and 180000 are recurrent strokes. ${ }^{3}$ In 2007, the overall mortality rate from stroke was 273000 , which makes stroke the third-leading cause of death in the United States. ${ }^{3}$ According to year book of the department of Medicine at DMCH (2009) 14.7\% of total admission was stroke patients.

Stroke is a complex disease that requires the efforts and skills of all members of the multidisciplinary team. ${ }^{4} \mathrm{~A}$ coordinated care of the stroke patient results in improved outcomes, decreased lengths of stay, and decreased costs. ${ }^{5}$ Stroke patient die off either due to the primary disease or due to complications. Medical management focus on the prevention of sub acute complications of stroke, including 
malnutrition, aspiration, pneumonia, dyselectrolytaemia, UTI, bowel or bladder dysfunction, DVT, pulmonary embolism, contractures, joint abnormalities, and skin breakdown. ${ }^{6}$ Electrolyte disturbances such as hypernatraemia or hyponatraemia, resulting from the syndrome of inappropriate antidiuretic hormone (SIADH), increase of brain natriuretic peptides (BNP), inappropriate fluid intake and loss, can lead to complications such as seizures or death. ${ }^{4,7}$ Most haemorrhagic stroke patients are presented with headache and vomiting. ${ }^{8}$ Vomiting is an important cause of dyselectrolytaemia. ${ }^{2}$ Complications like dyselectrolytaemia are more common in acute phase. ${ }^{4}$ In our country, there are many studies on stroke, its associated conditions and their effect on stroke patient's outcome, but a few studies on electrolytes disturbance in stroke patients has been done in our country, even outside. ${ }^{9}$ In this study, I attempted to find out the common electrolytes status in different type of acute stroke patients and their association with some common clinical presentation.

\section{Materials \& Method:}

In this descriptive cross sectional study, a total number of 100 randomly selected, clinically and CT proven acute stroke patients were studied from Jan 2010 to June 2010 at medicine units of Dhaka Medical College Hospital. Patient admitted within 48 hours of the onset of stroke with CT scan of the brain showing infarct or haemorrhage was enrolled for this study. Serum electrolytes level was done in all patients on admission. Association of electrolytes imbalance among acute stroke patient were identified and correlated. All data were collected in individual case record form. This was done by detailed history from patients or his / her relatives, complete physical examination and necessary investigations. Statistical analysis was carried out by using SPSS v16.0 Windows statistical software. Descriptive statistics were used for the interpretation of the findings. Informed and written consent obtained from all patients or their guardian. Formal Ethical Clearance was obtained from the Research Review Committee of Dhaka Medical College and Hospital.

\section{Result:}

Out of 100 patients $29 \%$ were in between 51-60 years age group \& $72 \%$ were male and $28 \%$ were female patients. According to CT scan findings majority $53 \%$ patients had Ischaemic stroke, 45\% Intracerebral haemorrhage (ICH) and only $2 \%$ had Subarachnoid haemorrhage (SAH). The low income group (63\%) comprises the major percentage of the patients in our study. $77.7 \%$ Haemorrhagic stroke and $75.4 \%$ Ischaemic stroke patients were hypertensive. $53 \%(p>0.05)$ of total acute stroke patient had dyselectrolytaemia. $62.22 \%$ of acute haemorrhagic stroke $(p<0.05) \& 43.39 \%$ of acute ischaemic stroke $(p>0.05)$ patients had dyselectrolytaemia. Among 100 acute stroke patient 49 (49\%) presented with vomiting from which $73.46 \%$ had dyselectrolytaemia which is statically significant $(\mathrm{p}=<0.01) .54(54 \%)$ presented with headache from which $74.07 \%$ had dyselectrolytaemia which is also statically significant $(\mathrm{p}=<0.01)$ (Table-I). In this study $66.66 \%$ haemorrhagic stroke and $28.30 \%$ ischaemic stroke patients had headache. $70.66 \%$ haemorrhagic and $30.23 \%$ ischaemic stroke patients had vomiting. 32\% of all stroke patients had hyponatraemia, Hyponatraemia was most common among haemorrhagic stroke patients $(19 \%)$ followed by ischaemic stroke patients (13\%). Only $4 \%$ of all stroke patients had hypernatraemia which was $3 \%$ of ischaemic stroke and $1 \%$ of haemorrhagic. Total $36 \%$ of all stroke patients had serum sodium imbalance during stroke. Among s. sodium imbalance hyponatraemia was most common (Table-II). $30 \%$ of all stroke patients had hypokalaemia. Hypokalaemia was most common among haemorrhagic stroke patients (19\%) followed by ischaemic stroke patients (11\%). Only $1 \%$ of all stroke patients had hyperkalaemia which was one of the patients of haemorrhagic stroke. Total $31 \%$ of all stroke patients had serum potassium imbalance during stroke. Among them hypokalaemia was most common (Table-III). In the table-IV, 16(16\%) had serum chloride imbalance and only $02(2 \%)$ had serum bicarbonate imbalance. The incidence of serum sodium, potassium, chloride and bicarbonate imbalances were higher in acute haemorrhagic stroke $(20,19,09 \& 02$ patients respectively) than acute ischaemic stroke (16, 12, $07 \& 0$ patients respectively).

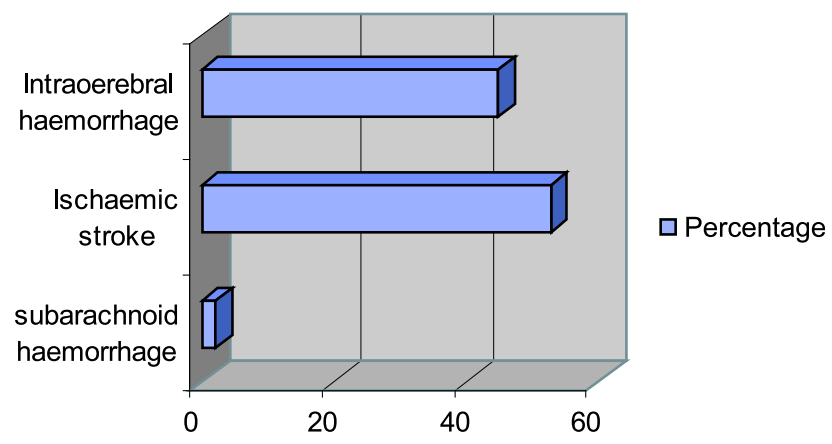

Fig.-1: Type of stroke according to CT scan findings

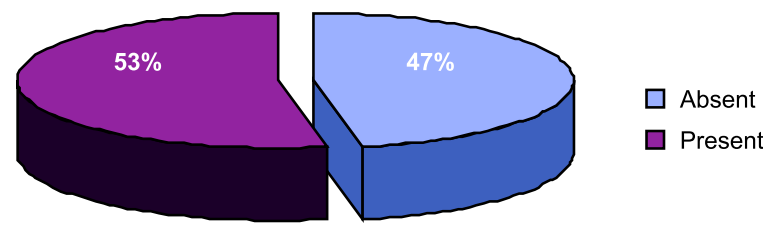

Fig.-2: Frequency of dyselectrolytaemia in acute stroke patients. 
Table-I

Association of different premonitory symptoms and dyselectrolytaemia in acute stroke patients.

\begin{tabular}{lcccc}
\hline $\begin{array}{l}\text { Premonitory } \\
\text { symptoms }\end{array}$ & $\begin{array}{c}\text { Dyselectrolytaemia } \\
\text { present (\%) }\end{array}$ & $\begin{array}{c}\text { Dyselectrolytaemia } \\
\text { absent (\%) }\end{array}$ & $\begin{array}{c}\text { Total } \\
(\%)\end{array}$ & $\begin{array}{c}p \\
\text { value }\end{array}$ \\
\hline Headache & $40(74.07)$ & $14(25.92)$ & $54(100)$ & $<0.001$ \\
Vertigo & $12(42.85)$ & $16(57.14)$ & $28(100)$ & $>0.05$ \\
Vomiting & $36(73.46)$ & $13(26.53)$ & $49(100)$ & $<0.01$ \\
Seizure & $2(28.57)$ & $5(71.42)$ & $07(100)$ & $>0.05$ \\
None & $6(30.00)$ & $14(70.00)$ & $20(100)$ & $>0.05$ \\
\hline
\end{tabular}

$\%:$ percentage,

Table-II

Frequency of serum sodium imbalance in stroke patient

\begin{tabular}{lcccc}
\hline Type of stroke & Normal S. Sodium (\%) & Hyponatraemia (\%) & Hypernatraemia (\%) & Total(\%) \\
\hline Haemorrhagic stroke & $27(27 \%)$ & $19(19 \%)$ & $1(1 \%)$ & $47(47 \%)$ \\
Ischaemic stroke & $37(37 \%)$ & $13(13 \%)$ & $3(3 \%)$ & $53(53 \%)$ \\
\hline Total & $64(64 \%)$ & $32(32 \%)$ & $4(4 \%)$ & $100(100 \%)$ \\
\hline
\end{tabular}

$\mathrm{n}$ : number of patient, s: serum, normal serum sodium : $(135-145 \mathrm{mmol} / \mathrm{l})$, hyponatraemia: $(<135 \mathrm{mmol} / \mathrm{l})$, hypernatraemia: $(>145 \mathrm{mmol} / \mathrm{l})$ Chi-square test $\left(\chi^{2}\right)$ was done to measure the level of significant ( $p$ value). $\chi^{2}=1.71$, df $=1, p=>0.05$, which revealed no significant association between hyponatraemia and type of stroke.

\section{Table-III}

Frequency of serum potassium imbalance in stroke patient

\begin{tabular}{lcccc}
\hline Type of stroke & Normal S. Potassium (\%) & Hypokalaemia (\%) & Hyperkalaemia (\%) & Total(\%) \\
\hline Haemorrhagic stroke & $28(28 \%)$ & $19(19 \%)$ & $1(1 \%)$ & $47(47 \%)$ \\
Ischaemic stroke & $41(41 \%)$ & $11(11 \%)$ & $0(0 \%)$ & $53(53 \%)$ \\
\hline Total & $69(69 \%)$ & $30(30 \%)$ & $1(1 \%)$ & $100(100 \%)$ \\
\hline
\end{tabular}

n: number of patient, s: serum, normal serum potassium : $(3.5-5 \mathrm{mmol} / \mathrm{l})$, hypokalaemia: $(<3.5 \mathrm{mmol} / \mathrm{l})$, hyperkalaemia: $(>5 \mathrm{mmol} / 1), \mathrm{Chi}-$ square test $\left(\chi^{2}\right)$ was done to measure the level of significant ( $p$ value). $\chi^{2}=5, \mathrm{df}=1, p=<0.05$, which revealed significant association between hypokalaemia and type of stroke.

Table-IV

Association of various type of dyselectrolytaemia in different type of acute stroke. $(n=100)$

\begin{tabular}{lccc}
\hline Various type of dyselectrolytaemia & Haemorrhagic stroke & Ischaemic stroke & Total $(\%)$ \\
\hline Sodium imbalance & 20 & 16 & $36(36)$ \\
Hyponatraemia & 19 & 13 & $32(32)$ \\
Hypernatraemia & 01 & 03 & $4(4)$ \\
Potassium imbalance & 19 & 12 & $31(31)$ \\
Hypokalaemia & 19 & 11 & $30(30)$ \\
Hyperkalaemia & 0 & 01 & $01(01)$ \\
Chloride imbalance & 09 & 07 & $16(16)$ \\
Hypochloraemia & 09 & 06 & $15(15)$ \\
Hyperchloraemia & 00 & 01 & $01(01)$ \\
Bicarbonate imbalance & 02 & 0 & $02(2)$ \\
Low bicarbonate & 02 & 0 & $02(02)$ \\
High bicarbonate & 00 & 0 & $00(00)$ \\
\hline
\end{tabular}

n:number of patient, \%:percentage, normal s. sodium : $(135-145 \mathrm{mmol} / 1)$, hyponatraemia: $(<135 \mathrm{mmol} / 1)$, hypernatraemia: $(>145 \mathrm{mmol} / 1)$, normal s. potassium : $(3.5-5 \mathrm{mmol} / 1)$, hypokalaemia: $(<3.5 \mathrm{mmol} / \mathrm{l})$, hyperkalaemia: $(>5 \mathrm{mmol} / \mathrm{l})$, normal s. chloride: $(96-110 \mathrm{mmol} / 1)$, hypochloraemia: $(<96 \mathrm{mmol} / \mathrm{l})$, hyperchloraemia: $(>110 \mathrm{mmol} / \mathrm{l})$, normal s. bicarbonate: $(24-30 \mathrm{mmol} / 1)$. 


\section{Discussion:}

Stroke incidence rises exponentially with increasing age. In this present study, maximum number of patients (29\%) were in between 51-60 years age group followed by (22\%) between $61-70$ years age group. The maximum number of male $(21 \%$ \& $16 \%)$ and female $(8 \% \& 6 \%)$ were also in the above age group respectively. Bevan $\mathrm{H}$ et al in his study of stroke also found similar picture. ${ }^{10} \mathrm{~A}$ hospital based study done in DMCH showed that only $1 \%$ occurred in $<20$ years and $26 \%$ in $20-45$ years and majority are above 45 years. ${ }^{11} 72 \%$ were male and $28 \%$ were female i.e., male incidence is $30 \%$ higher than female which coincide with international study. The present study coincides with the study of Chowdhury et al, and Kurtzke, where showed that frequency of stroke is $30 \%$ higher in men than women. ${ }^{12,13} \mathrm{CT}$ scan findings of the studied patients show that majority $53 \%$ patients had ischaemic stroke, $45 \%$ had intracerebral haemorrhage and only $2 \%$ had subarachnoid haemorrhage. This study similar with study of Alam B et al, they studied 1020 patients of stroke in DMCH. ${ }^{14}$ Higher rate of haemorrhagic stroke in this present hospital based study and previous Alam B et al study in DMCH may be due to the acute admission is more related to the haemorrhagic stroke. ${ }^{14}$

In this series $53 \%$ of our acute stroke patient had dyselectrolytaemia. $64.22 \%$ of acute haemorrhagic stroke $(p<0.05) \& 43.39 \%$ of acute ischaemic stroke $(p>0.05)$ patients had dyselectrolytaemia. In a study by Kusuda $\mathrm{K}$ et al, ${ }^{15}$ found that $52 \%$ haemorrhagic stroke $(p=<0.01)$ and $26 \%$ ischaemic stroke patients had dyselectrolytaemia ( $p=>0.05$ ). In this series the percentages of dyselectrolytaemia is a bit high, may be due to under developed acute management setup in our hospital. In this study (table-II), $32 \%$ of all stroke patients had hyponatraemia. Hyponatraemia was most common among haemorrhagic stroke patients (19\%) followed by ischaemic stroke patients (13\%). But chi-square test revealed no statistic significant association between hyponatraemia and type of stroke ( $p=>0.05$ ). Only $4 \%$ of all stroke patients had hypernatraemia which was $3 \%$ of ischaemic stroke and $1 \%$ of haemorrhagic stroke. Total $36 \%$ of all stroke patients had serum sodium imbalance during stroke. $30 \%$ of all stroke patients had hypokalaemia (tableIII). Hypokalaemia was most common among haemorrhagic stroke patients (19\%) followed by ischaemic stroke patients (11\%). chi-square test revealed significant association between hypokalaemia and haemorrhagic stroke $(\mathrm{p}=<0.05)$. Only $1 \%$ of all stroke patients had hyperkalaemia which was one of the two patients of haemorrhagic stroke. Total $31 \%$ of all stroke patients had serum potassium imbalance during stroke. 9\% haemorrhagic stroke and 6\% ischaemic stroke patient presented with hypochloraemia during stroke
( $p=>0.05) .2 \%$ haemorrhagic stroke patient presented with low bicarbonate level during stroke. In a study by Kusuda $\mathrm{K}$ et al, ${ }^{15}$ found that $34 \%$ acute stroke patients presented with serum sodium imbalance and $44 \%$ with serum potassium imbalance. Both hyponatraemia and hypokalaemia were more common among haemorrhagic stroke patients. All these findings are consistent with this present study.

In this study (table-I) $66.66 \%$ haemorrhagic stroke and $28.30 \%$ ischaemic stroke patients had headache. $70.66 \%$ haemorrhagic and $30.23 \%$ ischaemic stroke patients had vomiting. A study by Siddique AN et $\mathrm{al}^{16}$, headache was present in $60 \%$ patient of hemorrhagic stroke and $43.75 \%$ (35) patient of ischemic stroke. ${ }^{16}$ Vomiting was present in $75 \%$ cases of hemorrhagic stroke and $40 \%$ cases of ischemic stroke. ${ }^{16}$ In this study among 100 acute stroke patient 49 presented with vomiting from which $73.46 \%$ had dyselectrolytaemia which is statically significant $(\mathrm{p}=<0.01)$. 54 presented with headache from which $74.07 \%$ had dyselectrolytaemia which is also statically significant $(p=<0.01)$. So, dyselectrolytaemia were more who presented with headache and vomiting.

\section{Limitation of the study:}

The result of the present study should be interpreted in the light of the following limitations. First, the sample size was relatively small. Second, no long-term follow-up could be carried out. Third, the definite causes of these dyselectrolytaemia during acute stroke could not carryout. However, the main analysis of the present study was focused to find-out the electrolytes status and common electrolytes disturbances in different type of acute stroke patients and their correlation with different clinical presentations.

\section{Conclusion:}

Stroke is one of the foremost causes of morbidity, mortality and a socioeconomic challenge, more so in Bangladesh where health system including the rehabilitation is not within the reach of ordinary people. The results of the present study demonstrate that, in haemorrhagic stroke, the incidence of dyselectrolytaemia was more than ischaemic stroke and which were mostly hyponatraemia and hypokalaemia. Early detection and management of which can improve the overall outcome of stroke patients.

Conflict of Interest: We have no conflict of interest.

\section{References:}

1. Bergen DC. The world wide burden of neurologic disease. Neurology 1996; 47:21-50

2. Allen CMC, Lueck CJ, Dennis M. Neurological disease. In Nicholas AB (ed). Davidson's Principal and Practice of 
Medicine, $20^{\text {th }}$ edition. UK Churchill Livingstone Elsevier, 2006;1131-1235

3. Rosamond W, Flegal K, Furie K et al. Heart disease and stroke statistics: 2008 update: a report from the American Heart Association Statistics Committee and Stroke Statistics Subcommittee. Circulation 2008;117:25-146

4. Summers D, Leonard A, Wentworth D et al. Comprehensive Overview of Nursing and Interdisciplinary Care of the Acute Ischemic Stroke Patient. A Scientific Statement from the American Heart Association. Stroke 2009;40:2911-44

5. Alberts MJ, Hademenos G, Latchaw RE et al. Recommendations for the establishment of primary stroke centers: Brain Attack Coalition. JAMA 2000; 283: 3102-09

6. Langhorne P, Stott DJ, Robertson L et al. Medical complications after stroke: a multicenter study. Stroke 2000;31:1223-29

7. WHO STEPS Stroke Manual: the WHO STEP wise approach to stroke surveillance. STEPS Stroke Surveillance Manual (V2.1); 2006-05-09

8. Broderick J, Connolly S, Feldmann E et al. Guidelines for the Management of Spontaneous Intracerebral Hemorrhage in Adults 2007 Update. Stroke 2007;38:2001-23
9. Bhalla A, Sankaralingam S, Ruth Dundas R et al. Influence of Raised Plasma Osmolality on Clinical Outcome After Acute Stroke. Stroke 2000;31:2043-48

10. Bevan H, Sharma K, Bradly W. Stroke in young adults. Stroke 1990;21:382-86

11. Mohammad QD, Alam B, Habib M et al. Prevalence of stroke in Bangladeshi population-A population based study. JAFMC 2009;5(1):24-7

12. Chowdhury SZM. Study of risk factor in cerebrovascular disease- A study of 100 cases (Dissertation). BCPS 1991:48.

13. Kurzke JF. Epidemiology of cerebrovascular disease. In :P.Rowland L, editor. Merrtt's Neurology. Philadelphia: LLW;2000:135-76

14. Alam B, Mohammad QD, Habib M et al. Stroke evaluation risk factor. Bangladesh J of Neuroscience 1999;15(2):14-8

15. Kusuda K, Saku Y, Sadoshima S et al. Disterbances of fluid and electrolyte balance in patients with acute stroke. Nippon Ronen Igakkai Zasshi 1989;26(3):223-27

16. Siddique AN, Zannatun N, Mahbub MS et al. Clinical presentation and epidemiology of stroke -a study of 100 cases. J MEDICINE 2009;10:86-9 